\title{
LAS ESCUELAS LOW COST, SIN DOCENTES. LA PRIVATIZACIÓN DE LA EDUCACIÓN «POR DEFECTO»EN PAÍSES DE BAJOS INGRESOS $^{\mathrm{I}}$
}

\section{Low cost schools, without teachers. The privatization of education "by default» in low-income countries}

Leoncio Vega GiL

Universidad de Salamanca

Correo-e:lvg@usal.es

Andrea Martín García

Universidad de Salamanca

Correo-e: andreamartin@usal.es

Recibido: 22 de octubre de 2019

Envío a informantes: 3 de noviembre de 2019

Aceptación definitiva: 20 de abril de 2020

RESUMEN: El presente trabajo de investigación pretende realizar un análisis crítico y sociohistórico de la conformación y expansión de los modelos privados de educación, así como de las lógicas de la privatización de la educación en algunos países de bajos niveles de ingresos y de rentas. Son estos contextos los que están siendo utilizados por las nuevas formas de educación para experimentar, asentar y expandir modelos educativos de bajo coste y, por tanto, asequibles para las familias de bajos ingresos en contextos de precariedad económica y social. El trabajo se centra en el análisis de dos países y dos proyectos educativos. Se trata de Kenia, país epicentro de experimentación y expansión del modelo de BIA (Bridge International Academies), y Perú, en el que están proliferando

- El presente trabajo forma parte del Proyecto de Investigación de I+D concedido por la Consejería de Educación de la Junta de Castilla y León con el título de La sociedad «bien educada» de Castilla y León. Factores determinantes, acción política de mejora y retos sociales (2019-202I). Ref. sAoI7GI9. Programa de apoyo a Grupos de Investigación Reconocidos de las universidades públicas de Castilla y León (BOCyL, 9/ julio/2019). 
como las margaritas las Innova Schools (IE) de la mano de la corporación industrial y comercial más importante del país. Estos proyectos, apoyados incluso por las instituciones oficiales, son fruto de la ausencia de la red pública a causa de las limitaciones estructurales. La opción privada es más rentable económicamente y de mayor nivel de calidad que la ofrecida por la red oficial. Al final, estos proyectos ni son tan integrados, ni tan sociales, ni tan excelentes, ni tan pedagógicos como se presentan.

Palabras clave: privatización de la educación; educación por defecto; capitalismo pedagógico; bajo coste.

AвSTRACT: This research work tries to perform a critical and socio-historical analysis of the formation and expansion of private education models, as well as the logic of the privatization of education in some countries with low income. It is these contexts that are being used by new forms of education to experiment, establish and spread of lowcost and, therefore, affordable educational models for low-income families in contexts of economic and social vulnerability. The work focuses on the analysis of two countries and two educational projects. This is Kenya, the epicenter country of experimentation and spread of the BIA model (Bridge International Academies), and Peru, in which the Innova Schools (IS) are proliferating like daisies in the hands of the most important industrial and commercial corporation in the country. These projects, supported even by public institutions, are the result of the absence of the public network due to structural limitations and the high cost of the school. The private option is more economically profitable and of a higher level of quality of the official education offered. In the end, these projects are neither as integrated, nor as social, nor as excellent, nor as pedagogical as they are presented.

KEY WORDS: the privatization of education; default education; pedagogical capitalism; low income.

\section{Introducción}

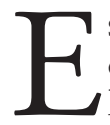

STE ES UN TRABAJO DE ANÁLISIS Y COMPRENSIÓN de uno de los nuevos itinerarios que sigue la privatización de la educación en distintos contextos internacionales. De especial incidencia en África y en América Latina. Se pretende analizar las condiciones, contextos y estructura pedagógica de proyectos escolares apoyados por corporaciones económicas y benefactores internacionales pensados para facilitar una nueva oportunidad educativa a familias y niños/niñas que, por el entorno social, económico y cultural en que se encuentran, no tendrían otra oportunidad educativa. También deben interpretarse en el sentido de «dejadez»o «abandono» de las estructuras públicas de los Estados que no son capaces de llegar a toda la ciudadanía. Estas nuevas formas de escolarización tienen especial significado y presencia en Kenia y en Perú, dos contextos distantes pero que coinciden en ciertas condiciones sociales y políticas. La conceptualización de estas formas de privatización de la educación tiene que ver con la "privatización por defecto», LFPs (Low Free Private School) (por sus siglas en inglés), privatización endógena/exógena (Verger, Fontdevila y Zancajo, 20ı6) y la gobernanza educativa (Vaillant, 20I2). En cuanto al estado de la cuestión no debemos pasar por alto los estudios publicados por J. Tooly (2015), que nos acercan tanto a las características de esta oferta privada de educación como al debate académico 
sobre su significado conceptual, social, político y económico, o también el estudio de L. Day Ashley et al. (20I4), que nos ayuda a comprender y valorar estos proyectos en países en vías de desarrollo en los que el impacto es más destacado.

El proceso metodológico nos conduce a un intento de aportación desde la perspectiva sociohistórica que incluye los contextos, los contenidos pedagógicos (actores, organización, competencias y rendición de cuentas) y la dimensión social de la privatización de la educación.

\section{Los contextos sociales}

Las formas o escenarios de privatización de la educación por defecto que pretendemos estudiar se concentran en Kenia, epicentro de las escuelas BIA (Bridge International Academies), aunque ya expandidas a otros países como Uganda, Liberia, Nigeria o India, y las Is (Innova Schools) en Perú. Dejamos para otra ocasión el estudio del modelo pay as you learn aplicado en las Omega Schools, de considerable impacto en Ghana (más de 38 escuelas y más de 20.000 estudiantes). El modelo LFPs no es exclusivo de Kenia y Perú; se ha expandido a otros entornos. Incluso otras iniciativas son de interés también como las Streetlight Schools (de Johanesburgo), que comienzan en 2013 y se consolidan en 20I6. La elección de BIA e Is se debe a tres razones que consideramos de interés. Por una parte, la amplia expansión institucional de ambos proyectos. Y, por otra, el impacto docente e investigador en el tejido académico universitario de gran atractivo para la política educativa, la educación comparada y la sociología de la educación. En tercer lugar, se trata de ejemplos escolares de la hiperglobalización a la que está siendo sometida la educación en el contexto internacional.

Kenia se constituye como república parlamentaria en 1964 tras la independencia del Imperio británico. La vida política está fragmentada y muy vinculada a las distintas etnias que conviven en el país (44). Tanto la vida política como la económica y educativa están muy relacionadas con la cultura anglosajona (USA y UK) y con la presencia de organismos internacionales como el Banco Mundial, el Fondo Monetario Internacional, el Banco Europeo de Inversiones, etc. Algunos indicadores pueden ser de interés para comprender el nivel de vida de la población keniana: el PIB per cápita se sitúa en 3.300 dólares PPA; la inflación ronda el 8\%; el índice de desarrollo humano (IDH) se coloca en 0,590 en 2017 , con una ligera mejora, pero en el puesto I42; y la tasa de alfabetización ronda el $78 \%$. La educación primaria es gratuita desde 2003 . A pesar de los grandes avances en los procesos de escolarización, sigue habiendo un volumen importante de niños y niñas sin cobertura educativa y la que se ofrece, especialmente en las escuelas estatales, presenta enormes carencias estructurales.

La tipología de las escuelas en Kenia presenta dos categorías: las escuelas públicas o gubernamentales y las escuelas privadas, aunque las segundas han ido evolucionando hacia escuelas comunitarias. Las escuelas públicas o gubernamentales son las más abundantes del país. La mayoría de estas escuelas se crearon mediante la iniciativa $\mathrm{Ha}$ rambee. La comunidad interviene en estas escuelas ayudando en la construcción de infraestructuras y otras aportaciones materiales. Las escuelas privadas dependen de empresas, sectores eclesiásticos o de organismos o asociaciones internacionales. Ambas escuelas son registradas por el Ministerio de Educación, Ciencia y Tecnología (MOEST) y tienen la obligación de cumplir con algunos requisitos y condiciones. Las 
escuelas comunitarias fueron creadas y financiadas por las propias comunidades que cuentan con el apoyo de las familias. Dado que el Gobierno no presta apoyo material, han de buscar sus propios patrocinadores. Son escuelas que han surgido como un medio viable para ampliar el acceso a la educación escolar, en el contexto de la Educación para Todos. Además, estas escuelas son una parte importante de los sistemas educativos en el África Subsahariana. Tienen características tanto de escuelas públicas como de los centros de educación no formal. Se sitúan fundamentalmente en asentamientos y barrios marginales. Solo en Nairobi se computan más de 350 escuelas comunitarias. El currículum que ofrecen es igual para escuelas públicas y privadas y distinto para los centros de educación no formal (Nyaomita, Njoroge, Oulai, Sankale y Mujidi, 2004).

En el caso de Perú, está en pleno proceso de expansión el modelo de las Escuelas Innova (las Innova Schools), surgido bajo el mandato del presidente Ollanta Humala (en 20I5). Se trata de un país con una población superior a los 3i millones de personas y una renta per cápita de algo más de 6.000 dólares, con más del $20 \%$ de la población en condiciones de pobreza. Y una inversión en educación que ronda el $4 \%$ del PIB. El sistema educativo se organiza y administra en tres niveles complementarios: central, regional y local; relacionados, en buena medida, desde una perspectiva jerárquica. No obstante, el sistema no funciona con los niveles competenciales que serían deseables. Los datos que facilita PISA indican esa precariedad en la educación peruana; la escala de competencias científicas se ha movido desde los 369 puntos en 2009 (la primera aparición en el programa) a 397 en 2015 , como se ve, con ligeros avances. En la de cultura lingüística de 36I puntos se ha mejorado hasta 398 en 20I5. Y en cultura matemática se ha pasado de 365 puntos a 387 en los mismos años. A nivel comparativo estos datos indican que está en los últimos puestos de la tabla. No obstante, si nos fijamos en el Informe sobre Desarrollo Humano (20I7), Perú se encuentra en el grupo de países con un IDH alto (en el puesto 88 con un índice de 0,750 sobre I,0); este índice combina los niveles de escolarización con la esperanza de vida y con el producto interior bruto y, por tanto, es un indicador general del bienestar humano. En 1997 se promulga la Ley de Inversión Privada en Educación, siendo A. Fujimori el presidente de la república. Esta normativa atraerá recursos a la creación de iniciativas particulares que vendrán a favorecer los procesos escolares y educativos de la sociedad peruana. Cabe destacar también la presencia en el contexto escolar de una considerable población indígena que llevó al país a una apuesta por la política intercultural y bilingüe a mediados de los años noventa. Esta política educativa bilingüe, enmarcada en el programa internacional de Educación para Todos (EFA), presenta ciertas dosis de contradicciones tanto a nivel docente como de participantes en los procesos educativos escolares (Valdiviezo, 2009).

\section{Fundamentación y diseño de las «escuelas low cost»}

La escuela privada en Kenia está en pleno proceso de expansión, especialmente en contextos de vulnerabilidad (Edwards, Klees y Wildish, 20I7). Muchas familias que viven en asentamientos y zonas marginales demandan para sus hijos una educación primaria gratuita que el Estado tiene la obligación de proporcionar, pero que no llega a los más necesitados. Debido a la escasa oferta que ofrece el Estado, comienzan a proliferar, tanto a través de iniciativas comunitarias (líderes comunitarios) como 
iniciativas particulares o societarias, las escuelas privadas que ofrecen educación y atención a precios asequibles. Además, el Gobierno está comprometido con las LFPs; las BIA iniciaron su andadura en 2007 en el marco de un Programa de Apoyo al Sector Educativo (2005-20Io) de la propia administración pública. También tienen la cobertura y apoyo de los organismos internacionales a través del Programa Educación para Todos. Según Bridge International Academies (2019), BIA o Puentes Internacionales son una sucursal de la compañía New Global Schools Inc. fundada en Delaware, USA, en el año 2007 por John Kimmelman, su esposa Shannon May y Phil Frei. Fundaron Bridge International Academies con el objetivo de trabajar de forma semejante a una franquicia de bajo coste con fines de lucro. BIA es una cadena de escuelas primarias y guarderías que buscan proporcionar acceso a una educación accesible de alta calidad para todas aquellas familias pobres que viven en países en vías de desarrollo (Kasturi y Lee, 20IO). BIA busca entregar una educación de alta calidad para todos los niños mediante la reducción del coste de los servicios tanto de profesorado como de infraestructura y material escolar. Este proceso se desarrolla a través del uso de economías de escala, con la ayuda de un monitoreo riguroso y un avanzado control de calidad que permite gestionar todos los datos de las escuelas Bridge (Kwauk y Perlman, 20I6). Los cofundadores del proyecto BIA serán reconocidos en 2014 como Emprendedores del Año por parte de la Fundación Schwab². También han recibido el premio World Innovation Summit for Education a la innovación educativa.

Las BIA están concentradas en ofrecer educación preescolar y educación primaria que facilitan la alfabetización numérica, científica y lingüística básicas, así como el pensamiento crítico (Kasturi y Lee, 20Io). Este es el caso también de las Is, cuyo germen está en 2005 y en la escuela San Felipe Neri de Lima promovida por Jorge Izusqui, que será, a partir de 20I0, el gerente general de las is en Perú. Es esta última fecha en la que el Grupo Intercorp decide invertir 150 millones de dólares para reconvertir las escuelas fundadas por Izusqui, nombrándolo gerente general en el gran proyecto pedagógico que llegarán a ser las IE; ya cuenta con más de 50 centros y una atención diaria a más de 40.000 alumnos. De esta forma, Izasqui no se desprendía de la propiedad de las escuelas. El mayor crecimiento de este proyecto privado de educación en Perú se produce entre 2015 y 20I6; se pasa de 18.535 alumnos a 24.572. También los beneficios se incrementarían un $40 \%$, como subraya la prensa. La ubicación geográfica de la red de is se centra en zonas costeras y en la capital del Estado y la zona metropolitana; están en 14 departamentos del país y acaban de abrir la primera escuela en México. Esta red privada de educación está mejorando sus indicadores, índices de calidad y

La Fundación Schwab es una plataforma dedicada a promover todos aquellos modelos, procesos y proyectos innovadores de carácter social que encuentren soluciones de apoyo a personas necesitadas y vulnerables de bajos ingresos. Una de sus apuestas más definidas es el emprendimiento social.

La figura principal de la red de centros de Innova Schools en Perú es Jorge Izasqui. Un emprendedor, autodidacta, ingeniero industrial formado en universidades privadas y entusiasta de la educación. Educador no de profesión sino de vocación. Su carrera educativa comienza con la dirección de un proyecto de capacitación docente en zonas rurales por encargo del IPAE (Instituto Peruano de Administración de Empresas), una organización empresarial (dirigida por el propio Jorge Izasqui entre 2009 y $201 \mathrm{I}$ ), en colaboración con el Ministerio de Educación. En 2005 funda un pequeño colegio en Los Olivos con el apoyo del padre Miguel Raneras Sánchez Pardo y Gonzalo Galdos (socio capitalista). En 2009 ya tenía abiertos 3 colegios privados con el apoyo financiero de Cofide (un banco de desarrollo de Perú fundado en 197I) (y no del BID al que había pedido ayuda). Ese año el Grupo Intercorp, a través de su presidente, se interesó por el proyecto de escuelas privadas y se inicia el gran proyecto de Is. 
aceptación social de la mano de dos órganos de acreditación y supervisión que están aliados en Perú: uno internacional, la Agencia de Acreditación de conocimiento, Estudiantes y Escuelas (AdvancED), con una experiencia de más de Ioo años y definida como fundación sin ánimo de lucro que persigue la excelencia. Se trata de una agencia global de acreditación y evaluación con una amplia oferta de servicios educativos y de aprendizaje con presencia en EE. UU. y en 70 países más. El aliado local es el SINEACE (Sistema Nacional de Evaluación, Acreditación y Certificación de la calidad de la Educación), una agencia peruana dedicada a acreditar y garantizar la calidad educativa. Los alumnos de la red is duplican los niveles competenciales de la media nacional. Y, según Infaboe (Perú), los alumnos de las is consiguen 29 puntos más en lectura que los alumnos de las escuelas públicas.

\section{La identidad pedagógica de BIA e INNOVA (IS)}

El análisis de estos proyectos escolares internacionales pasa por abordar el estudio de algunos parámetros o actores: destinatarios, docentes, las nuevas tecnologías de la información y la comunicación, la organización institucional y la participación de la comunidad social en la educación.

\section{I. School in a box}

School in a box es un proyecto «total» e «integrado» de educación. Incluye tanto la parte material de la escuela (construcción, mantenimiento, infraestructura, recursos, personal docente y de servicios, etc.) como la dimensión pedagógica (selección y formación de los profesores, definición e implementación del tiemplo escolar-calendario, semana, jornada escolar-) e, incluso, la organización y administración de la institución escolar. De tal forma que BIA examina, controla, supervisa y verifica toda la cadena de suministro (administrativo y pedagógico) del proceso escolar, incluso antes de haberse iniciado; de ahí la conceptualización de «school in a box». De tal forma que posee sus propios agentes inmobiliarios, sus empresas de construcción, sus fabricantes de recursos, sus diseñadores, sus creadores de software, su servicio de atención al cliente, sus propios uniformes, sus propios equipos de estudio sobre los terrenos, la comunidad y sus niveles de renta, el entorno, los estudios de mercado, etc. Es el modelo de BIA.

\subsection{La organización y gestión de las escuelas}

La creación y la expansión de los modelos educativos de privatización a bajo coste están en directa conexión o son producto de los procesos globales de organización de la educación que se asientan en políticas de descentralización de servicios y funciones al objeto de acercar más y mejor los servicios a los beneficiarios y liberar al Estado y los gobiernos de los compromisos directos con los procesos alfabetizadores y socializadores a través de la escolarización. Una descentralización que se considera beneficiosa tanto para la administración educativa como para las propias escuelas y la población del entorno (De Grauwe, Lugaz, Barasa, Tsepa, Kyabwe y Asiimwe, 20II). 
El Ministerio de Educación de Kenia comenzó en 2005 a financiar parte de los gastos de las LFPS, a través del Programa de Apoyo al Sector de Educación de Kenia. Este apoyo llegó a través de la iniciativa «Materiales de Instrucción», en el que las escuelas comenzaron a recibir financiación para pagar maestros y materiales, tales como tiza, borradores y libros. La cantidad asignada por alumno estaba destinada a equipar las escuelas con libros de texto clave sobre una base compartida de un libro por cada dos niños. Al frente de cada BIA se encuentra un gerente que reside en la zona, cuya misión principal es la dirección y control del proyecto pedagógico que gestiona siguiendo las indicaciones, criterios y directrices de la sede central de BIA. El seguimiento organizativo se realiza a través del Manual Operativo del cual se sirven los gerentes escolares, donde se incluye el procesamiento para administrar los recursos económicos, el personal de la escuela, la forma de interactuar con los estudiantes y los padres, el sistema central de procesamiento de nóminas, así como gastos, la evaluación estandarizada, las herramientas de evaluación y los recursos de evaluación para estudiantes y personal de la escuela (Kasturi y Lee, 2010). Además, cada uno de los administradores ha de seguir un programa de capacitación que incluye las lecciones y guías imprescindibles para maestros; un control estandarizado de las operaciones diarias, mensuales y anuales, así como coaching en habilidades financieras y de gestión. Finalmente, el administrador de la escuela recibe una bonificación mensual junto con su salario (Kasturi y Lee, 20Io). También en el caso de la red IE en Perú los administradores responsables de la organización de los centros escolares (directores de formación), una vez seleccionados de entre los que poseen estudios universitarios, han de cursar un programa específico de formación ofrecido por la red.

\subsection{Inscripciones y aceptación social (los destinatarios)}

Dada la precariedad y debilidad del modelo público de educación, se comprueba un aumento en el número de escuelas privadas, por compensación «o defecto». Estas aumentaron en Kenia un $4 \%$ en 2005 y un II \% en 2008. En el ámbito económico e ideológico estos proyectos privados de educación suelen estar vinculados a órdenes religiosas y grupos filantrópicos sin ánimo de lucro. El motivo por el cual muchas familias envían a sus hijos a escuelas privadas de bajo coste, como las BIA, se debe fundamentalmente a que creen que aunque el pago de las tarifas de estas escuelas es muy costoso y dificulta la subsistencia y el nivel de vida familiar, prefieren llevar a sus hijos a escuelas privadas puesto que en las escuelas públicas hay maestros que no están comprometidos, que se ausentan de sus clases, que no planifican las tareas pedagógicas, que se realizan actividades que no tienen contenido pedagógico, etc. Sin embargo, en las escuelas privadas son el mismo director de la escuela y su gerente los responsables ante las familias de cualquier actividad o problema que se manifieste (Kasturi y Lee, 20I0).

En 2014 se produce un alto crecimiento en el número de clases en las escuelas de Kenia debido al mayor número de alumnos de nuevo ingreso en las escuelas privadas que ofrecen alta calidad a un bajo coste. También se recogen en las pruebas internacionales, por primera vez, los resultados de alumnos de las BIA. Se obtienen resultados satisfactorios en los apartados de habilidades de lectura y matemáticas, superando el promedio del país. 
La conexión escuela/familia, en términos de rendición de cuentas, es muy estrecha en las escuelas Bridge; de tal forma que durante las 24 horas del día pueden ser informados de los procesos y prácticas pedagógicas del centro educativo. Además, se les pasan encuestas periódicas para tener conocimiento de su satisfacción o no de las prácticas formativas de sus hijos. Esta disponibilidad también es características de las Is; Izusqui informa que se comprometen a prestar atención en cualquier momento y a cualquier hora del día; sin secretarias y sin pedir citas.

En cada una de las escuelas ubicadas en zonas urbanas y marginales asisten aproximadamente un número de rooo estudiantes, incluidos en grados de preescolar/ pre-primaria (3-5 años) y primaria (6-II años). Cada clase cuenta con alrededor de un número de entre 55 y 65 alumnos por maestro. La cuota que tiene fijada BIA para los alumnos es de 4 dólares por mes/alumno $(3,5 \mathrm{I} €)$, que incluyen las horas lectivas y las sesiones de tutoría en las mañanas o en los sábados. En las IE se fijan tres tipos de cobros: ingreso, derecho de matrícula y la pensión o mensualidad.

En todo caso, el acceso a estas escuelas is no es directo, sino que consta de algunas pruebas que pretenden medir la capacidad intelectual de los futuros alumnos, sus rasgos emocionales, su comprensión lectora y la capacidad para resolver problemas. Y las cuotas de matrícula (incluidas las tres) no son tan low cost como podría pensarse dado que ronda una media de i85 dólares mensuales por todos los servicios educativos prestados por la red.

\subsection{El currículo y las TIC}

El Proyecto BIA se inicia en 2007. En el año 20ro se incluye el pago de tarifas mediante aplicaciones móviles, permitiendo a la escuela la anulación de operaciones corruptas y ofreciendo a las familias formas de pago más rápidas y útiles. A finales de 20 IO BIA inaugura Io escuelas en los barrios más pobres y marginales de la ciudad de Nairobi. En las diversas escuelas operan alrededor de 60 maestros y administradores escolares, gerente y director (BIA, 20I9). En el año 20II, se inauguran más escuelas en la ciudad de Nairobi. Además, este mismo año se inician diversas pruebas piloto en relación con el uso de un software para los teléfonos inteligentes que permitiría a los gerentes encargados de cada escuela obtener de manera detallada y mejor administrada la academia, así como el control pormenorizado del progreso y avance de cada alumno. En el año 20 I2 se incorpora en los teléfonos inteligentes una nueva aplicación denominada Academy Manager, que permite un control más avanzado y detallado de cada una de las operaciones y actividades realizadas en la escuela. En este mismo año, se realiza una mejora en las lecciones que se otorgaban a los maestros y a los alumnos, incluyendo mejor contenido, más minucioso y objetivo. Asimismo, las BIA incorporan a sus escuelas un proyecto denominado Academia en verde basado en el compromiso, respeto y concienciación de la importancia del medio ambiente, así como de su cuidado. En el año 20I3, se incorpora una tablet para que los maestros de las escuelas BIA tengan incluidas las planificaciones de las lecciones por actividad y fecha; la organización de las evaluaciones por alumno y contenido, y el seguimiento de los alumnos, las mejoras, retrasos, atrasos, comportamiento y actitud.

Todos los docentes, gestores, directores y coordinadores disponen de una tablet en las escuelas BIA; un recurso tecnológico que se convierte en un instrumento de 
información y formación, pero también de control y supervisión de las actividades y funciones laborales de todos y cada uno de los actores que intervienen en los procesos y prácticas educativas.

El modelo curricular de BIA es el oficial del sistema educativo que se fundamenta en el 4+4+4; primaria (6-IO), secundaria (II-I5) y universidad o educación superior (I622); con una previa educación preescolar de 3 a 5 años. El plan de estudio de educación primaria incluye inglés, kiswahili, matemáticas, ciencias, sociales, educación religiosa, artes creativas, educación física y habilidades para la vida. También en is se han de cumplir los parámetros curriculares oficiales. No obstante, tanto el proyecto como las familias insisten en la formación de líderes como una seña de identidad de las is; líderes no solo en términos de emprendedores, sino también en la vida social. La red de is ofrece toda la educación anterior a la educación superior y se enorgullecen de que el $84 \%$ de los mismos acceden a la universidad. En primer lugar, está la oferta inicial, la educación de la infancia de 3 a 5 años; la organización curricular y pedagógica de este nivel sigue las orientaciones y el asesoramiento de la U. de Berkeley para centrar su aportación metodológica en las experiencias, implicación de la familia y la participación activa del niño en su propio proceso de aprendizaje. La segunda sección ofrecida es la que corresponde a la educación primaria, en la que metodológicamente se trabaja con las aportaciones del modelo blended learning (aprendizaje, trabajo colaborativo y nuevas tecnologías), a través de dos estrategias organizativas: el group y solo learning. La tercera oferta es la que corresponde a la educación secundaria, que también se fundamenta en la metodología blended learning. En 2018 firmaron un proyecto de colaboración con Proyecto Zero de Harvard y ahora acaban de firmar la colaboración con Elesapiens Learning \& Fun SL, una empresa española con sede en Madrid, dedicada a la producción cinematográfica y de vídeo (con 7 años de experiencia) y que ofrece servicios educativos a centros escolares a través de su plataforma bilingüe de aprendizaje de ciencias (de 7 a I3 años); se trata de una librería de recursos educativos digitales para el aprendizaje de las ciencias a través de vídeos, juegos interactivos, láminas, infografía, etc.

\subsection{Los docentes (reclutamiento, formación y estatus)}

El maestro ejerce sus actuaciones pedagógicas en base al mandato y orientación de los recursos tecnológicos; en las tablets se encuentran los contenidos, las orientaciones metodológicas, el seguimiento de los avances formativos de los alumnos, las lecciones impartidas, las actividades pendientes, los progresos de los procesos de evaluación, los retrasos, los alumnos más lentos...; pero también los aspectos laborales del trabajo docente (entrada, salida, horas lectivas, etc.). Es decir, la tablet termina asumiendo la función docente y es también el instrumento de rendición de cuentas por excelencia y el docente se convierte en un mero técnico.

En este modelo de accountability los maestros reciben incentivos económicos en concepto de bonus por presentarse a clase, por las lecciones impartidas y, cómo no, según los resultados competenciales de los alumnos. Los incumplimientos laborales y el bajo rendimiento pueden ser penados hasta con el despido. No obstante, también son evaluados de forma periódica en relación con su desempeño docente; y las consecuencias pueden conllevar el aumento de incentivos. 
El proceso de selección de los maestros para las escuelas BIA exige previamente una certificación oficial y referencias, además de proceder de la comunidad y contexto en el que se ubica la escuela; debe haber superado la educación secundaria. Han de realizar una prueba competencial, así como una demostración práctica de sus capacidades pedagógicas como docente y una entrevista con responsables del proyecto BIA. Una vez elegidos los maestros, han de cursar y superar un programa de formación pedagógica intensiva en el Instituto Internacional de Formación (BITI), cuyos contenidos son tanto teóricos como prácticos, sobre la organización del aula, el plan de estudios y los diseños curriculares. Una vez contratados ya serán introducidos en procesos de inducción por parte del gerente de la escuela. En el caso de los docentes de la red IE un punto de partida es que posean estudios universitarios y, una vez seleccionados, han de cursar un programa específico de formación ofrecido por la propia red.

Los recursos didácticos que el proyecto BIA pone a disposición de los docentes pasan por las guías docentes (lecciones y asistencia), teléfonos inteligentes (asistencia de alumnos y maestros, pagos de matrícula y el monitoreo de instrucción), libros de historia (cuentos para que los alumnos se sientan identificados con los personajes), libros de tarea (controlar progreso) y señales (comunicaciones no verbales para las rutinas), además de pizarras (pizarras en miniatura para cada alumno), contadores de colores (tapas de botella para el reconocimiento de colores, habilidad motora fina y conteo), diez cuadros (cartones de huevos para el conteo en base io, contar, sumar, restar y uso de fracciones), libros de clase (al mismo tiempo que los libros de los maestros) y juguetes educativos como bloques de construcción, tarjetas de lazos, rompecabezas y muñecas de papel. Estos últimos pensados especialmente para los más pequeños. En primaria se completa con conjuntos de lectores (un libro de ficción y otro de literatura que enseñan fonética, comprensión lectora, interpretación, análisis y resolución de problemas), geobards (para realizar algoritmos, multiplicación, división, fracciones, geometría plana y álgebra básica), preparación para el examen nacional (ayuda en idiomas y problemas), kits de ciencia (brújulas, instrumentos meteorológicos, lupas, muestras de suelo, recipientes de plástico y experimentos) y mapas.

Bridge paga a sus maestros un promedio salarial de II4 \$ a I25 \$ por mes; una banda salarial menor que la ofrecida por el Gobierno en sus escuelas (I45 \$ a 175 \$). Además, el contrato de los maestros en Bridge incluye sanidad y pensión. Lo más importante es la regularidad en la percepción de los salarios, al contrario de lo que ocurre con los maestros de las escuelas oficiales que reciben sus dotaciones con varios meses de retraso. $\mathrm{Al}$ objeto de valorar el poder adquisitivo, hemos de tener en cuenta que unos zapatos en Nairobi cuestan 55,3I \$; un vestido 30,62 \$ y un billete en transporte público o,69 \$.

Las familias de los alumnos de las is ponen de manifiesto su satisfacción porque este proyecto pone en marcha metodologías activas, mucho más experimentales y mucho más personalizadas que en las escuelas oficiales. Sus hijos han de ser exitosos no solo en la universidad, sino también en la vida. La red is se define metodológicamente a través del constructivismo. Selecciona a sus docentes a través de entrevistas que realiza el gerente de la red. Los docentes en los colegios is no ejercen de enseñantes, sino que la mayor parte del tiempo escolar (el $70 \%)$ lo dedican a debates en torno a temas o preguntas y el otro $30 \%$ al trabajo pedagógico con los recursos tecnológicos. No obstante, son conscientes de que un gran problema pedagógico radica en las deficiencias de la formación de los profesores; por ello tienen previsto abrir durante el año en curso una escuela de formación de docentes. 


\subsection{El tiempo escolar}

En las escuelas Bridge se trabaja para conseguir el éxito educativo reforzando las prácticas y los tiempos educativos si fuera necesario. En principio, como norma, el calendario se compone de 45 semanas al año y la semana escolar consta de 6 días. En cuanto a la jornada, esta se inicia a las 7:30 y finaliza a las 17:00 horas; los sábados se termina una hora antes.

\section{Una lectura sociológica de la privatización de la educación}

Un análisis que pretendemos realizar en cuatro dimensiones: contextual, promotores, tipología de las familias y la implicación del Estado. En relación con la primera variable tenemos que poner de manifiesto que las LFPS en general, y las BIA e Is en particular, se ubican en contextos fundamentalmente urbanos en el marco de las grandes ciudades como hemos comprobado en el apartado anterior; dejan fuera, por tanto, los contextos rurales (de gran importancia demográfica en ambos países). Esto plantea un problema de accesibilidad a la educación/escolarización, como han puesto de manifiesto organizaciones internacionales como la Campaña Mundial por la Educación (CME), que afecta al «derecho universal a la educación» (Walker, 20I6).

Las LFPs están apoyadas, patrocinadas y protegidas por poderosos actores económicos internacionales. Bridge cuenta con grandes promotores y patrocinadores como Bill Gates, IFC International Finance Corporation, World Bank Group, la Agencia Británica de Desarrollo Internacional y Pearson Education, entre otros. El epicentro de BIA es Kenia; no obstante, el proyecto se ha expandido a países como Liberia, Uganda, Nigeria, Reino Unido o India, como hemos puesto de manifiesto. Los cofundadores de BIA son Phil Frei ${ }^{4}$, Shannon May5 y Jay Kimmelman 6 . Otras

4 Phil Frei es de origen canadiense. Sus idiomas calificados son el alemán, el español y el inglés. Obtuvo diversos reconocimientos y premios. Por un lado, fue galardonado por el Annual Design Review - Best of Category ID Magazine en el año 200o. Por otro lado, por el Fellowship Interval Research Corporation en el año I999 y, finalmente, por el Fellowship LEGO en el año 1998. Estudió en Massachusetts, en el Institute of Technology BS, Mechanical Engineering, durante los años I994-1998. A continuación, estudió MIT Media Lab y finalmente en Massachusetts, Institute of Technology MS, Media Technology durante 1998 hasta el año 200o. Phil Frei formó parte de los siguientes grupos: UCC grupos, MIT Massachusetts Institute of Technology Alumni, Global MIT Enterprise Forum y finalmente en MIT Media Lab Alumni (Bridge International Academies, 2019).

5 Shannon May es licenciada por la Universidad Magna con Cum Laude en Estudios Sociales de Harvard y doctorada en Antropología por la uc Berkeley. Realizó estudios sobre ecología y desarrollo económico. Trabajó como asesora de diversas organizaciones internaciones relacionadas con el diseño y desarrollo de ciudades sostenibles. Ha ejercido como maestra en escuelas de China y de Estados Unidos. Finalmente, se encarga de liderar la expansión territorial de la cadena de escuelas Bridge. Además, asume la investigación, así como las relaciones gubernamentales, el desarrollo de los negocios, la supervisión del marketing y la estrategia de marca.

6 Jay Kimmelman es cofundador y director ejecutivo de BIA. Es el proveedor de servicios de aprendizaje en la nube; líder en el mundo para estudiantes que viven con menos de $2 \$$ por persona y día. Es líder en el campo de la innovación educativa. Anteriormente fundó y dirigió Edusoft, compañía de software educativo en escuelas públicas de EE. uU. Tras la venta de Edusoft fundó Bridge con el objetivo de proporcionar una educación a los niños cuyas familias viven con menos de $2 \$$ por día. Fue galardonado y recibió el premio a su B.A. en informática e ingeniería eléctrica, Magna cum laude, Phi Beta Kappa, de la Universidad de Harvard. 
aportaciones personales de interés en BIA son: Prabhakar Sunder, director financiero de las escuelas; Sean Geraghty, director académico de las escuelas BIA; Olu Babalola, director de BIA en Nigeria; Ranjit Koshi, director de BIA en la India; Griffin Asigo, director de BIA en Liberia; Kent McNeill, jefe de tecnología de BIA. Otros grupos de «inversores» son: Bill y Melinda Gates Foundation (Seattle, 200o); CDC "Commonwealth Development Corporation"Investment Works (depende de UK); Chan Zuckerberg (acciones en Facebook); IFC (International Finance Corporation); World Bank Group (el Banco Mundial invirtió mucho dinero para ampliar la red internacional de BIA); Jasmine Social Investments (fundación privada creada por Sam Morgan en 2006, con sede en Nueva Zelanda); Khosla Ventures (empresa de capital/riesgo estadounidense); Learn Capital (empresa de capital/riesgo creada por Paul Strange en el año 20Io, destinada a financiar a emprendedores); LGT Impact Ventures (fundación privada con sede en Nueva Zelanda); NEA (firma de capital/riesgo estadounidense); Nigeria Sovereign Investment Authority (administra un fondo de riqueza); Novastar Ventures; ON (Omidyar Network) (firma filantrópica creada en 2004 por Pierre Omydar); Pan African Investment Co (empresa de inversión privada con sede en N. York, creada por Richard Perarson y Ronald Lauder); Pearson (multinacional británica creada en I998, que ofrece servicios educativos); Pershing Square Foundation (compañía estadounidense de gestión de fondos ubicada en Nueva York y creada por Bill Ackman).

En el caso de las is en Perú los fines «lucrativos» y la «educación como industria» están más claros y definidos que en Kenia. El presidente de is es el mismo que el de Intercorp, Carlos Rodríguez Pastor, el hombre más rico del país. Este holding empresarial está conformado por una tupida e integrada red de más de 60 corporaciones empresariales dedicadas a los más variados temas económicos, desde banca a centros comerciales pasando por seguros, universidades, agencias inmobiliarias, grupos de capital/riesgo, etc., con expansión en Hong Kong y Shanghái. No obstante, a pesar de que el coste parece considerable dado que una familia aporta I50o \$ al año para que su hijo estudie en un colegio Is, el gerente general de la red considera el proyecto low cost porque lo compara con lo que cuesta al sector público la escolarización de un alumno de secundaria que se cifra en $\mathbf{3} 300$ \$. Es, por tanto, como él mismo afirma, un proyecto educativo al alcance de la clase media peruana. En ambos proyectos, los fines educativos son más que lucrativos. El alumno ha de pagar una cuota de 185 dólares mensuales (incluye ingreso, derecho de matrícula y mensualidad), en un país con un salario mínimo situado en 255 dólares.

La tercera variable de análisis se refiere a la implicación/participación de la comunidad social en estos proyectos LFPS. La gestión económica de las escuelas BIA está centralizada; los padres pagan la matrícula utilizando el programa M-PESA, un servicio de banca móvil que les permite realizar el pago de las cuotas a través del teléfono móvil o en una sucursal de equity bank. También el salario de los maestros se gestiona de manera centralizada a través del teléfono móvil. ¿Por qué las familias de bajos ingresos se decantan por las opciones privadas de educación? Para Tooley, Dixon y Stanfield (2008), las familias eligen las escuelas BIA por tres razones, entre otras. Por un lado, la calidad de la educación ofrecida: menor número de alumnos que en las oficiales; procesos y prácticas pedagógicas «individualizados»; docentes comprometidos, y rendición de cuentas. En segundo lugar, la cuestión económica: tarifas o cuotas sumamente flexibles y adaptadas a las condiciones vitales de las familias. También 
el tema de los uniformes; la permisividad que ofrece BIA a las familias para la obtención de un único uniforme en sus escuelas frente a la obligatoriedad de las escuelas del Gobierno que imponen el uso mínimo de al menos dos uniformes, así como el chándal para la materia de educación física. Las familias ponen en cuestión la red oficial de escuelas por sus carencias pedagógicas: maestros sin formación (y ausentes en muchos casos), intervenciones sin planificación pedagógica y actividades y tareas sin contenido pedagógico. Es decir, desde la percepción de las familias, estos proyectos privados de educación ofrecen mayor calidad y a un menor coste que el ofrecido por las escuelas oficiales. No obstante, las familias no son tan vulnerables como parece. En Perú han de aportar I85 dólares al mes y en Kenia 4 dólares; teniendo en cuenta el PIв y el coste de la vida, como hemos recogido anteriormente, no se trata de familias de bajos ingresos, sino de grupos sociales medios en términos de renta.

En párrafos anteriores hemos podido comprobar que estos proyectos privados de educación vienen apoyados por los gobiernos y los Estados a través de distintos programas, lo que puede interpretarse como un reconocimiento explícito de las debilidades estructurales para ofrecer una red oficial de escolarización universal. Pero también puede ser interpretado en el sentido de que el Estado ofrece educación a un menor coste por la delegación de funciones que, según la interpretación de la educación como bien público, debería ofrecer a la comunidad social al objeto de garantizar la equidad, la inclusión, la igual calidad, la accesibilidad y, por tanto, hacer efectivo el derecho a la educación que estos países tienen aceptado a nivel internacional.

\section{Conclusiones}

En el contexto africano Kenia se ha convertido en uno de los laboratorios experimentales de la «nueva escuela» debido a la gran demanda social de educación, las deficiencias de la red oficial de escolarización y la oferta low cost de educación puesta en marcha por actores corporativos internacionales con interés lucrativo (directo o indirecto).

Un tema central de la calidad de la educación y del derecho a la educación es el referido a la calidad de la función docente. Hemos podido comprobar que esta es una de las debilidades de los proyectos LFPs. Los docentes son sustituidos por la tecnología (tablets, móviles, ordenadores...). Los maestros son técnicos que se encargan del seguimiento de la planificación de las prácticas pedagógicas diseñadas por la Sede Central de BIA. En el caso de la red is de Perú también las nuevas herramientas tecnológicas vienen a sustituir la función docente; como veíamos hacen mucho hincapié en la metodología blended learning (con asesoramiento, recursos didácticos y apoyo de universidades como las de Berkeley o Harvard y empresas tecnológicas dedicadas a la educación como la EL and Fun sL radicada en Madrid). Los maestros no son docentes, enseñantes o profesores, son supervisores. Estamos, tal vez, ante la vuelta al unimetodismo; el docente como técnico y/o supervisor y los «maestros ignorantes» [siguiendo el método de J. J. Jacotot (1770-I840) (Rancière, I987)]. Además, o incluso, estos docentes están mal pagados (peor que en la red pública) y con considerables deficiencias formativas.

En términos de calidad de la educación y derecho a la misma, la CME se fija en la accesibilidad, la equidad, la inclusión y el bien público. La pretendida calidad de la 
educación, tanto en BIA como en Is, a pesar de la prensa y de sus propios resultados (siempre mejores que en la red pública) (el 84\% de los alumnos de las is consigue acceder a la Universidad), deja bastante que desear, teniendo en cuenta estándares occidentales. Ya poníamos de manifiesto el problema de la accesibilidad, tanto por razones geográficas como económicas. También otros indicadores nos ayudan a comprender y valorar la misma, como el elevado número de alumnos por clase/docente que en BIA llega hasta 65; las condiciones materiales (espacios, energía, mobiliario, etc.) y didácticas tampoco presentan los niveles de calidad deseables. Y si nos fijamos en la figura del docente esta imagen se distorsiona aún más. En BIA son reclutados con estudios secundarios, eso sí, de la propia comunidad, y después se los somete a un programa de formación para recibir unas dotaciones salariales que oscilan entre II4 y I25 dólares, mientras que los maestros oficiales perciben unas dotaciones, en Kenia, que oscilan entre 145 y 175 dólares. En todo caso, un poder adquisitivo muy bajo socialmente. En las is los docentes requieren estudios universitarios, pero sin formación pedagógica inicial; después de ser reclutados han de seguir un programa formativo de la propia red. La propia red se plantea como reto mejorar la formación de profesores. La equidad también se resiente tanto por cuestiones referentes a las cuotas, como por la distinta accesibilidad como por la contextualización, a pesar del esfuerzo compensador a través del ofrecimiento de becas, descuentos por razones de hermanos o según rendimiento o, incluso, el aplazamiento del pago de las cuotas. En estas condiciones está en juego la educación como bien público.

Uno de los grandes activos de estos proyectos LFPs es la rendición de cuentas, favorecida y apoyada en las nuevas tecnologías de la información y comunicación; se podría hablar, teniendo en cuenta el contexto, de inflación de las TIC. Se trata de la conexión fluida y permanente con las familias, del seguimiento del uso de los recursos, del seguimiento y supervisión tanto de las cuotas como de las dotaciones salariales de los docentes, de los mecanismos de evaluación de competencias en términos de resultados y difusión social de los mismos; de las campañas de imagen, etc. Así como de su gestión también «privada»: cada centro está en manos de un gerente que vela por el buen funcionamiento y uso de los recursos. Y la información del uso y rendimiento de los recursos que es facilitada a los actores patrocinadores.

Además, teniendo en cuenta su carácter «inversor», su ubicación contextual, su estructura organizativa y de gestión, su carácter «compensador» de las deficiencias del sistema público de educación, su estrategia gerencial de redición de cuentas, sus patrocinadores y promotores e, incluso, sus formas pedagógicas eminentemente tecnológicas, etc., bien podríamos calificar esta estrategia de privatización de la educación como el «nuevo capitalismo pedagógico» al interpretar el capital humano infantil como bien de inversión e industria y, cómo no, a las familias que sustentan las ofertas formativas, siguiendo la conceptualización de Sheila Slaughter y Larry Leslie (I999 y 2009).

Por tanto, estos proyectos de LFPS hemos de circunscribirlos a la libre elección de centro, tendencia internacional en expansión; es decir, la libertad de enseñanza y, también, a la globalización; o mejor la hiperglobalización. Hasta estas últimas décadas los proveedores de servicios educativos privados venían siendo proveedores o agentes locales. Pero la tendencia ha cambiado; ahora se trata de agencias, fundaciones, corporaciones, grupos, etc., de carácter internacional; son los nuevos proveedores de servicios educativos privados que suplen o compiten con las redes públicas de educación (Verger, Fontdevila y Zancajo, 2016). 


\section{Bibliografía}

Ashley, L. D.; McLoughlin, C.; Aslam, M.; Engel, I.; Wales, I.; Rawal, S.; Batley, R.; Kingdon, G.; Nicolai, S. y Rose, P. (20I4). The role and impact of private schools in developing countries: a rigorous review of the evidence. Final report. Education Rigorous Literature Review, April 20I4, I-74. Recuperado el I9 de abril de 2020, de file://C:/Users/ Leoncio/Downloads/private-schools-full-report.pdf.

Asley, L. D.; Mcloughlin, C.; Aslam, M. y Wales, J. (20I4). The role and impact of private schools in developing countries. Education Rigorous Literature Review.

De Grauwe, A.; Lugaz, C.; Barasa, T.; Tsepa, M.; Kayabwe, S. y Asimmwe, W. (20iI). Strengthening local actors: the path to decentralizing education: Kenia, Lesotho, Uganda. IIEP.

Edwards, B.; Klees, S. A. y Wildish, J. (20I7). Dynamics of Low-Fee Private Schools in Kenya: Governmental Legitimation, School-Community Dependence, and Resource Uncertainty. Teachers College Record, IIg(7), I-42.

Heyneman, S. P. y Stern, J. M. B. (20I3). Low cost private schools for the poor: What public policy is appropriate? International Journal of Educational Development. Recuperado el 20 de noviembre de 2019, de https://dialnet.unirioja.es/servlet/articulo?codigo=4736419.

Kasturi, V. y Lee, K. (20Io). Bridge International Academies: A School in a Box. Harvard Business School Case, (9), 5II-064. Recuperado el 20 de noviembre de 2019, de https://www.bridgeinternationalacademies.com/wp-content/uploads/20I8/o8/Case-Study-Harvard-Business-School-2oio.pdf.

Kwauk, C. y Perlman, J. (2016). Bridge International Academies. Delivering Quality Education at a low cost in Kenya, Nigeria, and Uganda. Center for Universal Education at Brookings. Recuperado el 2 de noviembre de 20I9, de http://www.bridgeinternationalacademies. com/wp-content/uploads/2016/og/Brookings-Millions-Learning-case-study.pdf.

Nyaomita, E.; Njoroge, J.; Oulai, D.; Sankale, J. y Mujidi, L. (2004). Community Schools in Kenya. Case study on community participation in funding and managing schools. International Institute for Educational Planning. Recuperado el is de octubre de 2019, de http:// www.unesco.org/iiep.

Rancière, J. (1987). Le maître ignorant. Cinq leçons sur l'émancipation intellectuel. Fayard.

Slaugter, S. H. y Leslie, L. (I999). Academic Capitalism: Politics, Policies and the Entreprenaurial university. J.H. University.

Slaughter, S. H. y Rhoades, G. (2009). Academic Capitalism and the New Economy. Markets, State, and Higher Education. J.H.U. Press.

Tooly, J. (20I5). Low-cost private schools: what we need to know, do know, and their relevance for education and development. En S. McGrath y Q. Gu (eds.) Routledge Handbook of International Education and Development (pp. 226-240). London: Routledge.

Tooley, J.; Dixon, P. y Stanfield, J. (2008). Impact of Free Primary Education in Kenya. A case study of private schools in Kenya.

Tooly, J. y Longfield, D. (20I5). The role and impact of private schools in developing countries: a response to the DFID-Commissioned. Rigorous Literature Review. Pearson. Recuperado el is de abril de 2020 , de eprint.ncl.ac.uk.

Vaillant, D. (20I2). La gobernanza educativa y los incentivos docentes: los casos de Chile y Uruguay. Revista Uruguaya de Ciencia Política, I(2I), I-I8.

Valdiviezo, L. A. (2009). "Don't You Want Your Child to Be Better than You»? Enacting Ideologies and Contesting Intercultural Policy in Peru. En F. VAvrus y L. Bartlett (eds.) Critical Approaches to Comparative Education (pp. I47-I62). Palgrave Macmillan.

Verger, A.; Fontdevila, C. y Zancajo, A. (20I6). The Privatization of Education. A Political Economy of Global Education Reform. New York: Teachers College Press.

Walker, J. (20I6). Beneficios privados, pérdidas públicas: por qué el impulso de las escuelas privadas de bajo coste está desencaminando la educación de calidad. Shaharazad Abuel-Ealeh. 
